\title{
Folliculitis, CTCAE
}

National Cancer Institute

\section{Source}

National Cancer Institute. Folliculitis, CT CAE. NCI Thesaurus. Code C143209.

A disorder characterized by inflammation or infection of the hair follicles. 